PROCEEDINGS OF THE AMERICAN MATHEMATICAL SOCIETY

Volume 124, Number 2, February 1996

\title{
A ZFC EXAMPLE (OF MINIMUM WEIGHT) OF A LINDELÖF SPACE AND A COMPLETELY METRIZABLE SPACE WITH A NONNORMAL PRODUCT
}

\author{
L. BRIAN LAWRENCE
}

(Communicated by Franklin D. Tall)

\begin{abstract}
We give an example as indicated in the title where the weight (i.e., the minimum cardinality of a base for the topology) of the product is the smallest uncountable cardinal.
\end{abstract}

\section{Introduction and the Main Theorem}

History. Throughout this paper the term "space" abbreviates "Hausdorff regular topological space". Is there a normal space and a metric space with a nonnormal product? The answer is yes in ZFC and is due to E. A. Michael ([6], 1963). (ZFC denotes the standard set of axioms for set theory.) Let $\mathbb{P}$ and $\mathbb{R}$ denote respectively the irrationals and the reals with their usual topologies; and let $\mathbb{M}$ denote the Michael line which is the refinement of $\mathbb{R}$ obtained by isolating each irrational point. In Michael's example, $\mathbb{M}$ is the normal space (and moreover hereditarily paracompact) and $\mathbb{P}$ is the metric space. Note that the weight of $\mathbb{M}$, and therefore the product, is $\mathbf{c}$ (c denotes the cardinality of the continuum of real numbers). Is there a ZFC example of a normal space with weight $\aleph_{1}$ (the smallest uncountable cardinal) whose product with $\mathbb{P}$ is nonnormal? (This question is due to Ronnie Levy.) It is not possible in ZFC to construct a Lindelöf space with both $\aleph_{1}$ weight and a nonnormal product with $\mathbb{P}$ (Lawrence, [5], 1990).

Define a Michael space to be a Lindelöf space whose product with $\mathbb{P}$ is nonnormal. The existence of a Michael space follows from both the Continuum Hypothesis (Michael, [7], 1971; first mentioned in a footnote of [6]) and Martin's Axiom (K. Alster, [1], 1990). Is there a Michael space in ZFC? This is Michael's problem and is equivalent to the existence in ZFC of a Lindelöf space and a separable completely metrizable space with a nonnormal product (see [5]).

Suppose we drop the requirement for completeness on the metrizable factor. In the 1963 paper, Michael also gave a ZFC example of a Lindelöf space and a separable metric space with a nonnormal product. The Lindelöf space is the refinement of $\mathbb{R}$ obtained by isolating each point in a Bernstein set, and the metric space is the Bernstein set as a subspace of $\mathbb{R}$. A subset of $\mathbb{R}$ is Bernstein if both it and its

Received by the editors November 24, 1992 and, in revised form, March 14, 1994.

1991 Mathematics Subject Classification. Primary 54B10; Secondary 54D20, 54E50.

Key words and phrases. Product, Lindelöf, completely metrizable, normal, Michael line, Michael space, Continuum Hypothesis, Martin's Axiom. 
complement intersect every Cantor set. Note that a Bernstein set necessarily has cardinality $\mathbf{c}$, so as before, the weight of the refinement is c. However, a nonnormal product of weight $\aleph_{1}$ can be constructed by replacing the Bernstein set in each factor of Michael's example with any subset of a Bernstein set having cardinality $\aleph_{1}$ (this was pointed out to the author by Gary Gruenhage).

Now suppose that we drop separability but hold on to completeness. This too can be achieved in ZFC, and moreover, under the constraint of $\aleph_{1}$ weight (which, as we noted above, is impossible if $\mathbb{P}$ is the metric space).

Main Theorem. There is a ZFC example of a Lindelöf space and a completely metrizable space where the product is nonnormal and has weight $\aleph_{1}$.

Remark. The reader familiar with logic will note that some of the proofs in this paper could be replaced by arguments using elementary submodels; we have avoided that approach however, in order not to limit the accessibility of the paper.

\section{Definitions And notation}

Spaces. Let $D=\omega_{1}$ with the discrete topology, and let $L=\omega_{1}+1$ where each point other than $\omega_{1}$ is isolated and a basic neighborhood at $\omega_{1}$ is of the form $\left[\alpha, \omega_{1}\right]$ for some $\alpha<\omega_{1}$; so we have that $L$ is Lindelöf and $D$ is a subspace of $L$. (Of course, the space $L$ is the analogue, for the Lindelöf property, of the one-point compactification of $D$.)

Our metrizable space, to be denoted by $M$, is the Tychonoff product of $\omega$-many copies of $D$. Recall that $\mathbb{P}$ is homeomorphic to the Tychonoff product of $\omega$-many copies of a countably infinite discrete space; so $M$ is the $\aleph_{1}$ version of $\mathbb{P}$. Note that $M$ is completely metrizable and has weight $\aleph_{1}$. (The space $\mathbb{P}$ (resp., $M$ ) is sometimes called the Baire space of weight $\aleph_{0}\left(\right.$ resp., $\left.\aleph_{1}\right)$.)

We define our Lindelöf space to be the following subspace $X$ of the Tychonoff product of $\omega$-many copies of $L$; so each factor of our example, and therefore the product itself, is homeomorphic to a subspace of ${ }^{\omega} L$. Let $X=\left\{\langle\alpha, f\rangle \in L \times{ }^{\omega} L\right.$ :

$\alpha=\operatorname{Lub} \operatorname{Ran} f\}$ (Lub abbreviates least upper bound, and $\operatorname{Dom} f$ and $\operatorname{Ran} f$ denote respectively the domain and the range of $f$ ). Note that for every ordered pair $\langle\alpha, f\rangle \in X, \alpha=\omega_{1}$ iff $\exists n \in \omega\left[f(n)=\omega_{1}\right]$. Let $Y=\left\{\langle\alpha, f\rangle \in X: \alpha=\omega_{1}\right\} ; Y$ is closed in $X$. As a subspace of $L \times{ }^{\omega} L, X$ has weight $\aleph_{1}$.

Both the full product, ${ }^{\omega} L$, and the difference, ${ }^{\omega} L \backslash M$, are Lindelöf (Theorem 1); moreover, each of the products, ${ }^{\omega} L \times M$ and $\left({ }^{\omega} L \backslash M\right) \times M$, is paracompact (Theorem 3); so the nonmetrizable factor in our counterexample was necessarily constructed as a nonclosed subspace of ${ }^{\omega} L$ in which the intersection with $M$ is unbounded (with respect to the pointwise partial order on $M$ ).

Let $E=\left\{f \in{ }^{\omega} L: \forall n \in \omega[f(2 n) \in D]\right\}$. Note that $E$ is homeomorphic to ${ }^{\omega} L \times M$. (Incidentally, by Theorem $3, E$ provides an example of a dense paracompact subspace of ${ }^{\omega} L$ which is neither Lindelöf nor a union of open-closed Lindelöf subspaces (as in the case of ${ }^{\omega} L \times{ }^{n} D$ for $n<\omega$ ).)

Finite sequences. Let $R=\{\sigma: \exists n \in \omega(\sigma:[0, n] \rightarrow L)\}$; let $S=\{\sigma \in R: \forall n \in$ $\omega$ with $2 n \in \operatorname{Dom} \sigma[\sigma(2 n) \in D]\}$; and let $T=\{\sigma \in R$ : $\operatorname{Ran} \sigma \subseteq D\}$.

Basic open sets. Suppose $\langle\sigma, \alpha\rangle \in R \times \omega_{1}$. Then define $A(\sigma, \alpha)=\prod_{n \in \omega} \Delta_{n}$ where: for each $n \in \operatorname{Dom} \sigma, \Delta_{n}=\{\sigma(n)\}$ if $\sigma(n) \in D$, and $\Delta_{n}=\left[\alpha, \omega_{1}\right]$ if $\sigma(n)=\omega_{1}$; and for each $n \notin \operatorname{Dom} \sigma, \Delta_{n}=L$. We define $B(\sigma, \alpha)$ similarly except that $R \times \omega_{1}$ is replaced with $S \times \omega_{1}$ in the choice of $\langle\sigma, \alpha\rangle$, and for each $n \notin \operatorname{Dom} \sigma, \Delta_{n}=D$ if $n$ 
is even and $\Delta_{n}=L$ if $n$ is odd. Let $\mathcal{A}=\operatorname{Ran} A$, and let $\mathcal{B}=\operatorname{Ran} B$. Then $\mathcal{A}$ is a base for ${ }^{\omega} L$, and $\mathcal{B}$ is a base for $E$.

A subcollection $\mathcal{U} \subseteq \mathcal{A}$ (resp., $\mathcal{B}$ ) is minimal provided that for each $\sigma \in R$ (resp., $S$ ), there is at most one $\alpha \in \omega_{1}$ with $A(\sigma, \alpha)$ (resp., $\left.B(\sigma, \alpha)\right) \in \mathcal{U}$. Note that for every $\mathcal{U}$, there is a minimal subcollection $\mathcal{U}^{\prime} \subseteq \mathcal{U}$ with $\bigcup \mathcal{U}^{\prime}=\bigcup \mathcal{U}$ (simply choose for each $\sigma$ the least $\alpha$ ).

\section{Normal SUbSpaces (of the full Product ${ }^{\omega} L$ )}

Continuous functions. Suppose $\mathcal{U}$ is a minimal subcollection of $\mathcal{A}$ (resp., $\mathcal{B}$ ) such that $\mathcal{U}$ covers ${ }^{\omega} L \backslash M$ (resp., $\left.\left({ }^{\omega} L \backslash M\right) \cap E\right)$. Define $\mathcal{V}=\mathcal{V}(\mathcal{U})$ on $\omega_{1}$ by $\mathcal{V}_{\alpha}=\{A(\sigma, \beta)$ (resp., $\left.B(\sigma, \beta)) \in \mathcal{U}: \operatorname{Ran} \sigma \subseteq[0, \alpha) \cup\left\{\omega_{1}\right\}\right\}$. Then:

(1) each $\mathcal{V}_{\alpha}$ is countable (by taking $\mathcal{U}$ to be minimal);

(2) for $\alpha<\beta, \mathcal{V}_{\alpha} \subseteq \mathcal{V}_{\beta}$; and

(3) for $\alpha$ a limit ordinal, $\mathcal{V}_{\alpha}=\bigcup_{\beta<\alpha} \mathcal{V}_{\beta}$.

Define $\Phi=\Phi(\mathcal{U})$ on $\omega_{1}$ by $\Phi_{\alpha}=\operatorname{Lub}\left\{\beta \in \omega_{1}: \beta=\alpha\right.$, or $\exists \sigma \in R$ (resp., $\sigma \in S$ ) $\left[A(\sigma, \beta)(\right.$ resp., $\left.\left.B(\sigma, \beta)) \in \mathcal{V}_{\alpha}\right]\right\}$.

Then:

(1) $\Phi: \omega_{1} \rightarrow \omega_{1}$ is continuous (with respect to the order topology) and monotone nondecreasing;

(2) for each $\alpha \in \omega_{1}, \Phi_{\alpha} \geq \alpha$; and

(3) for each $\alpha \in \omega_{1}, \mathcal{V}_{\alpha}$ is a cover of $\left\{f \in \bigcup \mathcal{U}\right.$ : $\left.\operatorname{Ran} f \subseteq[0, \alpha) \cup\left[\Phi_{\alpha}, \omega_{1}\right]\right\}$ (resp., $\left\{f \in \bigcup \mathcal{U}: \forall n \in \omega\left([f(2 n)<\alpha] \&\left[f(2 n+1)<\alpha\right.\right.\right.$ or $\left.\left.\left.\left.f(2 n+1) \geq \Phi_{\alpha}\right]\right)\right\}\right)$.

To prove the third property holds, take the case $\mathcal{U} \subseteq \mathcal{A}$ and choose $f \in \bigcup \mathcal{U}$ with $\operatorname{Ran} f \subseteq[0, \alpha) \cup\left[\Phi_{\alpha}, \omega_{1}\right]$. Define $g: \omega \rightarrow L$ by $g(n)=f(n)$ if $f(n)<\alpha$, and $g(n)=$ $\omega_{1}$ otherwise. By our covering hypothesis on $\mathcal{U}$, there exists $\sigma \in R$ with $\sigma \subseteq g$ and $\beta \in \omega_{1}$ such that $g \in A(\sigma, \beta) \in \mathcal{U}$; by the definition of $g, \operatorname{Ran} \sigma \subseteq[0, \alpha) \cup\left\{\omega_{1}\right\}$, which implies $A(\sigma, \beta) \in \mathcal{V}_{\alpha}$; and finally by the definition of $\Phi, \beta \leq \Phi_{\alpha}$, so $f$ is also in $A(\sigma, \beta)$. An analogous argument handles the case $\mathcal{U} \subseteq \mathcal{B}$.

Note that the first two properties imply that the fixed point set for $\Phi$ (i.e., $\left.\left\{\alpha \in \omega_{1}: \Phi_{\alpha}=\alpha\right\}\right)$ is a closed unbounded subset of $\omega_{1}$.

Theorem 1. Each of ${ }^{\omega} L$ and ${ }^{\omega} L \backslash M$ is Lindelöf.

Proof. Let $\mathcal{U}$ be a minimal subcollection of $\mathcal{A}$ which covers either ${ }^{\omega} L$ or ${ }^{\omega} L \backslash M$. Let $\alpha$ be a fixed point of $\Phi=\Phi(\mathcal{U})$. Then for $\mathcal{V}=\mathcal{V}(\mathcal{U}), \mathcal{V}_{\alpha}$ is a countable cover of either ${ }^{\omega} L$ or ${ }^{\omega} L \backslash M$ depending upon the initial choice of $\mathcal{U}$.

Theorem 2. Every unbounded subset of $M$ (with respect to the pointwise partial order) has a limit point in ${ }^{\omega} L \backslash M$.

Proof. Let $N$ be an unbounded subset of $M$. For each $n \in \omega$, let $\operatorname{Proj}_{n}: M \rightarrow D$ be the projection map defined by $\operatorname{Proj}_{n}(f)=f(n)$. We'll construct the limit point, $g$, and an auxiliary sequence, $\left\langle J_{n}: n \in \omega\right\rangle$ of nested subsets of $N$, by recursion on $n$.

Since $N$ is unbounded, we can choose $m \in \omega$ and an uncountable $N^{\prime} \subseteq N$ so that the restriction $\operatorname{Proj}_{m} \mid N^{\prime}$ is 1-1. We can now begin our recursion. Each stage has two cases. Case 1 for the first stage: $\operatorname{Proj}_{0}\left[N^{\prime}\right]$ is countable. Then choose $g(0)$ so that $J_{0}=\left\{f \in N^{\prime}: f(0)=g(0)\right\}$ is uncountable. Case 2: $\operatorname{Proj}_{0}\left[N^{\prime}\right]$ is uncountable. Then choose $J_{0} \subseteq N^{\prime}$ so that for every $\alpha \in \operatorname{Proj}_{0}\left[N^{\prime}\right]$, there is exactly one $f \in J_{0}$ with $f(0)=\alpha$. In this case, let $g(0)=\omega_{1}$. At stage $n$, for $n>0$, use the above construction with $J_{n-1}$ in place of $N^{\prime}$ and $\operatorname{Proj}_{n}$ in place of $\operatorname{Proj}_{0}$. 
Corollary (to Theorems 1 and 2). The space $X$ is Lindelöf.

Proof. The result follows from two facts. First, the subspace $Y \subseteq X$ is homeomorphic to ${ }^{\omega} L \backslash M$, and is therefore Lindelöf by Theorem 1. Second, by Theorem 2, for every open set $U$ containing $Y:\left\{\alpha \in \omega_{1}: \exists f \in M[\langle\alpha, f\rangle \in X \backslash U]\right\}$ is bounded; and therefore, $X \backslash U$ is homeomorphic to a subspace of $\mathbb{P}$.

Theorem 3. Each of ${ }^{\omega} L \times M$ and $\left({ }^{\omega} L \backslash M\right) \times M$ is paracompact.

Proof. Let $\mathcal{U}$ be a minimal subcollection of $\mathcal{B}$ which covers either $E$ or the subspace $E^{\prime}=\left\{f \in E: \exists n \in \omega\left[f(n)=\omega_{1}\right]\right\}$. Let $\Phi=\Phi(\mathcal{U})$, and let $\theta$ be the fixed point set for $\Phi$. Let $\mathcal{V}=\mathcal{V}(\mathcal{U})$. By the third property of $\Phi$, for each $\alpha \in \theta, \mathcal{V}_{\alpha}$ covers $F_{\alpha}=\left\{f \in E\left(\right.\right.$ resp., $\left.\left.E^{\prime}\right): \forall n \in \omega[f(2 n)<\alpha]\right\}$.

With respect to the subspace topology inherited from $\omega_{1}$, let $I(\theta)$ be the set of all isolated points in $\theta$. For each $\alpha \in I(\theta)$, let $\mathcal{W}_{\alpha}$ be the set of all $B(\sigma, \alpha) \in \mathcal{B}$ such that:

(1) $\operatorname{Ran} \sigma \subseteq[0, \alpha) \cup\left\{\omega_{1}\right\}$;

(2) $\exists V \in \mathcal{V}_{\alpha}[B(\sigma, \alpha) \subseteq V]$; and

(3) $\forall \tau \in S$ with $\tau \subseteq \sigma\left(\exists V \in \mathcal{V}_{\alpha}[B(\tau, \alpha) \subseteq V] \Rightarrow \tau=\sigma\right)$.

Then $\mathcal{W}_{\alpha}$ is a basic open pairwise disjoint refinement of $\mathcal{V}_{\alpha}$ with $\bigcup \mathcal{W}_{\alpha}=\bigcup \mathcal{V}_{\alpha}$.

We claim that for each $\alpha \in I(\theta), \bigcup_{\beta<\alpha}\left(\bigcup \mathcal{W}_{\beta}\right)$ is closed (resp., closed in $\left.E^{\prime}\right)$. Fix $\alpha$, and choose $f$ in the complement of the corresponding union. Let $\gamma=\operatorname{Lub}\{\beta \in$ $I(\theta): \beta<\alpha\}$. Then $f \notin F_{\gamma}$. Let $m \in \omega$ with $f(2 m) \geq \gamma$. Define $\sigma \in S$ by $\sigma \subseteq f$ and $\operatorname{Dom} \sigma=[0,2 m]$. Then for each $\beta<\alpha$ and each $B(\tau, \beta) \in \mathcal{W}_{\beta}$, we have the following implications: (1) $B(\sigma, \gamma) \cap B(\tau, \beta) \neq \emptyset \Rightarrow \operatorname{Dom} \tau \subseteq[0,2 m-1]$; and therefore, (2) $B(\sigma, \gamma) \cap B(\tau, \beta) \neq \emptyset \Rightarrow B(\sigma, \gamma) \subseteq B(\tau, \beta)$.

The following collection is an open (resp., open in $E^{\prime}$ ) pairwise disjoint covering (resp., covering of $\left.E^{\prime}\right) \quad$ refinement of $\mathcal{U}: \quad\left\{B(\sigma, \alpha) \backslash \bigcup_{\beta<\alpha}\left(\bigcup \mathcal{W}_{\beta}\right)\right.$ (resp., $\left.\left.\left[B(\sigma, \alpha) \backslash \bigcup_{\beta<\alpha}\left(\bigcup \mathcal{W}_{\beta}\right)\right] \cap E^{\prime}\right): \alpha \in I(\theta) \& B(\sigma, \alpha) \in \mathcal{W}_{\alpha}\right\}$.

\section{NONNORMAL SUBSPACES}

Closed sets. Define $C$ on $T$ by $C(\sigma)=\left\{\left\langle\omega_{1}, f\right\rangle \in Y: f \supseteq \sigma\right\}$.

Fact 1. Each value of $C$ is a closed set in $X$.

Fact 2. For every $f \in M, \bigcap_{\sigma \subseteq f} C(\sigma)=\emptyset$.

Fact 3. Suppose that for every $\sigma \in T, U(\sigma)$ is an open set in $X$ containing $C(\sigma)$. Then there exists $f \in M$ such that $\bigcap_{\sigma \subseteq f} U(\sigma) \neq \emptyset$.

Proof of Fact 3. Recursively choose a sequence $\left\langle\sigma_{k}: k \in \omega\right\rangle$ in $T$ so that $\sigma$ and the corresponding sequence $\left\langle g_{k}: k \in \omega\right\rangle$ in ${ }^{\omega} L$ (defined by $g_{k} \supseteq \sigma_{k}$ and, for each $\left.n \notin \operatorname{Dom} \sigma_{k}, g_{k}(n)=\omega_{1}\right)$ satisfy the following conditions:

(1) $\operatorname{Dom} \sigma_{k}=[0, k]$;

(2) $\sigma_{k}$ is strictly increasing;

(3) $\sigma_{k+1} \supseteq \sigma_{k}$; and

(4) $\alpha=\sigma_{k+1}(k+1)>\sigma_{k}(k)$ is chosen so that for some $\tau \in R$ with $\sigma_{k} \subseteq \tau \subseteq g_{k}$, if $V=\left[\alpha, \omega_{1}\right] \times A(\tau, \alpha)$, then $\left\langle\omega_{1}, g_{k}\right\rangle \in V \cap X \subseteq U\left(\sigma_{k}\right)$.

Let $f=\bigcup_{k \in \omega} \sigma_{k}$, and let $\beta=\operatorname{Lub} \operatorname{Ran} f$. Then $\langle\beta, f\rangle \in \bigcap_{k \in \omega} U\left(\sigma_{k}\right)$. 
Remark. The strategy that underlies the proof of Theorem 4 is an application of T. C. Przymusiński's characterization theorem on normality in the product of a normal space and a metric space (see his Handbook of Set-Theoretic Topology article: [8]).

Theorem 4. The product $X \times M$ is nonnormal (and, by Theorem 3, is homeomorphic to a subspace $G$ of ${ }^{\omega} L$ which can be written as a union of $\aleph_{1}$-many pairwise disjoint closed (in $G$ ) paracompact subsets).

Proof. Let $f: T \rightarrow M$ be a 1-1 function such that for all $\sigma, \tau \in T$ with $\sigma \neq \tau$ : if $n$ is the least index on which $\sigma$ and $\tau$ disagree, or $\tau$ is a proper extension of $\sigma$ and $n$ is the least index in $(\operatorname{Dom} \tau) \backslash(\operatorname{Dom} \sigma)$, then $n$ is also the least index on which $f_{\sigma}$ and $f_{\tau}$ disagree. Note that $\operatorname{Ran} f$ (as a subspace of $M$ ) is discrete in itself, while $(\operatorname{Ran} f)^{\prime}$ (the set of all limit points of $\operatorname{Ran} f$ ) is homeomorphic to $M$.

Let

$$
H=\bigcup_{\sigma \in T}\left(C(\sigma) \times\left\{f_{\sigma}\right\}\right) \text { and } K=X \times(\operatorname{Ran} f)^{\prime} .
$$

Then $H$ and $K$ are disjoint closed sets (Facts 1 and 2) which cannot be separated by disjoint open sets (Fact 3).

Remark. Our use of Przymusiński's characterization theorem illustrates an alternative to the classical strategy discovered by Michael for constructing nonnormal products; Theorem 4 can also be proved using Michael's prototypical choice of disjoint closed sets which cannot be separated: $Y \times M$ and the diagonal $\{\langle\langle\alpha, f\rangle, f\rangle: f \in$ $M \& \alpha=\operatorname{Lub} \operatorname{Ran} f\}$.

Theorem 5. For every $f \in{ }^{\omega} L \backslash M$, each of the subspaces ${ }^{\omega} L \backslash\{f\}$ and $X \backslash\left\{\left\langle\omega_{1}, f\right\rangle\right\}$ is nonnormal.

Proof. Let $w=f^{-1}[D]$, and choose $n_{0}$ arbitrarily in $\omega \backslash w$. Define $\lambda \in \omega_{1}$ by $\lambda=0$ if $w=\emptyset$, and otherwise by $\lambda=\operatorname{Lub}\{f(n): n \in w\}$. For each $\alpha \geq \lambda$, define $g_{\alpha} \in{ }^{\omega} D$ by $g_{\alpha}(n)=f(n)$ if $n \in w$, and $g_{\alpha}(n)=\alpha$ if $n \notin w$. Then in the subspace ${ }^{\omega} L \backslash\{f\}$, $\left\{h: h \neq f \& h\left(n_{0}\right)=\omega_{1}\right\}$ and $\left\{g_{\alpha}: \alpha \geq \lambda\right\}$ (resp., in $X \backslash\left\{\left\langle\omega_{1}, f\right\rangle\right\}, Y \backslash\left\{\left\langle\omega_{1}, f\right\rangle\right\}$ and $\left.\left\{\left\langle\alpha, g_{\alpha}\right\rangle: \alpha \geq \lambda\right\}\right)$ are disjoint closed sets which cannot be separated by disjoint open sets.

For a proof in the case of ${ }^{\omega} L \backslash\{f\}$, first note that a basic open set about $g_{\alpha}$ is determined by an integer $k\left(g_{\alpha}\right)$ (agreement with $g_{\alpha}$ on the initial segment $\left[0, k\left(g_{\alpha}\right)\right]$ ). Given $k$, choose $m$ so that $\left\{\alpha \geq \lambda: k\left(g_{\alpha}\right)=m\right\}$ is cofinal in $\omega_{1}$. Then any function which agrees with $f$ on $w \cap[0, m]$ and constantly takes the value $\omega_{1}$ on $[0, m] \backslash w$ belongs to the closure of the union of the open sets defined by the function $k$.

\section{REFERENCES}

1. K. Alster On the product of a Lindelöf space and the space of irrationals under Martin's Axiom, Proc. Amer. Math. Soc., 110 (1990), 543-547. MR 90m:54012

2. - Some remarks concerning the Lindelöf property of the product of a Lindelöf space with the irrationals, Topology Appl. 44 (1992), 19-25. MR 93g:54013

3. K. Alster and G. Gruenhage, Remarks on the product of Lindelöf spaces (to appear).

4. Amer Beslagic, Normality in products, Ann. New York Acad. Sci. 705 (1993), 17-46. MR 95f: 54013

5. L. B. Lawrence, The influence of a small cardinal on the product of a Lindelöf space and the irrationals, Proc. Amer. Math. Soc., 110 (1990), 535-542. MR 90m:54014

6. E. A. Michael, The product of a normal space and a metric space need not be normal, Bull. Amer. Math. Soc., 69 (1963), 375-376. MR 27:2956 
7. _ Paracompactness and the Lindelöf property in finite and countable cartesian products, Compositio Math., 23 (1971), 199-214. MR 44:4706

8. T. C. Przymusiński, Products of normal spaces, Handbook of Set-Theoretic Topology (K. Kunen and J. E. Vaughan, eds.), North-Holland, Amsterdam, 1984, pp. 781-826. MR 86c:54007

Department of Mathematics, George Mason University, Fairfax, Virginia 22030-4444 